\title{
2 RESEARCH OF PROCESSES OF PRODUCING MATERIALS BY TECHNICAL POWER LOADING SYSTEMS
}

\author{
I. Nazarenko, Ye. Mishchuk, M. Kyzminec, \\ S. Oryshchenko, O. Fedorenko, S. Tsepelev
}

ABSTRACT

An increase in the effective working process of the crusher is achieved when it operates in the vicinity of resonance, i.e. at a high value of the coefficient of resonant amplification of oscillations. The optimal values of the amplitude of the oscillations of the masses have the greatest values, provided that the coefficient of regulation of elasticity in frequency $n$ is in the range of $0.6 \leq n \leq 1$. Within the frequency range of $14.5 \mathrm{~Hz}<f<26 \mathrm{~Hz}$, material is effectively crushed. The numerical values of the coefficient are established, which determine the rational ratios of vibration masses. The studies carried out and their results indicate that the main stresses causing the destruction of the material are shear stress. Analytical equations are obtained that reflect the picture of energy consumption for the destruction of the material. Such studies are planned as a continuation of the topic under consideration through experimental studies and consideration of several members of the series and optimization of the parameters of crushing machines. The proposed approach to studying the energy characteristics of material destruction in the crusher chamber with a guaranteed zone of stability of parameters in the resonance zone can be used for other processes. Such processes include the destruction of materials during dynamic cutting of soils, processing of materials in mills and sorting of materials, which are widely used in Ukraine abroad. An algorithm and method for calculating the main parameters of a vibration crusher have been developed.

\section{KEYWORDS}

Vibration crusher, material, grinding, physical, mathematical model, equation, resonance, parameters, destruction, stability zone, stress, deformation, energy consumption.

\subsection{SELECTION AND JUSTIFICATION OF PHYSICAL AND MATHEMATICAL MODELS OF TECHNICAL SYSTEMS FOR MATERIALS GRINDING PROCESSES}

Vibration jaw crushers are a relatively new direction in the development of crushing technology, which are highly energy efficient and are used in various industries, including construction. The most promising of the known vibration jaw crushers are crushers with two movable jaws and crushers with a heavy body and a pendulum. On the other hand, the creation of such machines is hindered by the severity of providing a resonant mode of operation in which the efficiency of crushers increases significantly. 
Let's consider a model of a working fluid in terms of interaction with a machine. Let's consider the layout of the elements of the vibration system under study in the following order (Fig. 2.1): vibration exciter - machine body - working medium - striker. Let's take: $m$ - total mass of the machine; $c$ - coefficient of elasticity of elastic connections; $m_{m}$ - mass of the material; $F_{0}(t)$-driving force; $x_{n}$ - movement of individual elements of the crusher structure; $D$ - average diameter of the input material.

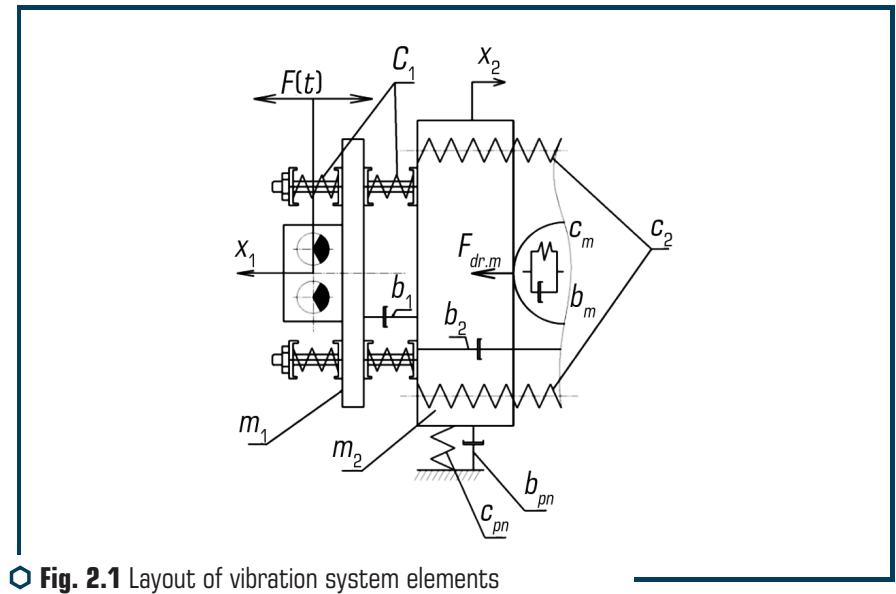

The equation of motion of our system will be an equation of the form [1]:

$$
\frac{\partial^{2} x}{\partial u^{2}}=\frac{1}{c^{2}} \frac{\partial^{2} x}{\partial t^{2}}
$$

where $c$ - speed of the wave propagating in the medium.

The solution to equation (2.1) will be the dependence [1]:

$$
x(u, t)=\left(A \cos \frac{\omega}{c} v+B \sin \frac{\omega}{c} v\right) \sin \omega t .
$$

Coefficients $A$ and $B$ are determined by the boundary conditions and, by their physical content, reflect the vibration amplitudes in the corresponding contact zones.

Forces acting on the contact between the crushing plate and the material:

$$
-F_{c r . m}=F_{0} \sin \omega t-\left(m_{1} \ddot{x}_{1}+2 x_{1} c_{1}-x_{2} c_{1}+m_{2} \ddot{x}_{2}+x_{2} c_{1}-x_{1} c_{1}+2 c_{2} x_{2}-2 c_{2} x_{3}+x_{3} c_{f}\right),
$$

where $F_{c r . m .}$ - reaction force of the material from the action of the crushing jaw lequal to the crushing force with the opposite sign); 


$$
F_{0} \sin \omega t-\left(m_{1} \ddot{x}_{1}+2 x_{1} c_{1}-x_{2} c_{1}+m_{2} \ddot{x}_{2}+x_{2} c_{1}-x_{1} c_{1}+2 c_{2} x_{2}-2 c_{2} x_{3}+x_{3} c_{f}\right)
$$

- crusher motion equation.

On the other hand, the expression for the reaction of the material to the crushing plate can be written as follows:

$$
F_{c r . m}=\sigma S=E S\left(\frac{\partial x}{\partial u}\right)
$$

Equating expressions (2.3) and (2.4) let's obtain:

$$
E S\left(\frac{\partial x}{\partial u}\right)=F_{0} \sin \omega t-\left(\begin{array}{l}
m_{1} \ddot{x}_{1}+2 x_{1} c_{1}-x_{2} c_{1}+m_{2} \ddot{x}_{2}+ \\
+x_{2} c_{1}-x_{1} c_{1}+2 c_{2} x_{2}-2 c_{2} x_{3}+x_{3} c_{o n}
\end{array}\right) .
$$

Let's find the unknown derivatives $\partial x / \partial u$ and $\ddot{x}=\partial x^{2} / \partial^{2} t$ by differentiating equation (2.2):

$$
\begin{aligned}
& \frac{\partial x_{2}}{\partial u}=\left(-A \frac{\omega}{c} \sin \frac{\omega}{c} v+B \frac{\omega}{c} \cos \frac{\omega}{c} v\right) \sin \omega t, \\
& \frac{\partial x_{2}^{2}}{\partial^{2} t}=-\left(A \cos \frac{\omega}{c} v+B \sin \frac{\omega}{c} v\right) \omega^{2} \sin \omega t .
\end{aligned}
$$

To find the displacements of the first and third masses included in equation (2.5), let's use the solution in the form of the following functions:

$$
\left\{\begin{array}{l}
x_{1}[t]=A_{1} \sin \omega t \\
x_{3}[t]=A_{3} \sin \omega t
\end{array}\right.
$$

Thus, an equation can be derived to determine the vibration amplitudes of the contact zone:

$$
\begin{aligned}
& B_{2}=A_{2} \tan \left(\frac{r \omega}{c}\right), \\
& A_{2}=-\frac{A_{1} c_{1}-2 A_{3} c_{2}+A_{3} c_{o n}-F_{0}-\omega^{2} A_{1} m_{1}}{2 c_{2}-\omega^{2} m_{2}+E S\left(\frac{\omega}{c}\right) \tan \left(\frac{r \omega}{c}\right)} .
\end{aligned}
$$

As can be seen from equation (2.10), the effect of the material on the dynamics of the mouse is determined by a parameter $E S(\omega / c) \tan (r \omega / c)$ that can be turned into the following form [1]: 


$$
m_{m} \omega^{2}\left(\frac{\operatorname{tg} \psi}{\psi}\right)
$$

where $m$ - the mass of the material.

The ratio $(\operatorname{tg} \psi / \psi)=\gamma$ is called the coefficient of added mass. It takes into account the difference between the mass $m_{m}$ and its static value $m_{m s}[1]$.

When designing any machine, great attention should be paid to the rational selection of kinematic parameters. The kinematic parameters mainly include the geometric characteristics of the machine as a whole and its individual parts.

One of the features in the design of vibration crushers is the presence of vertical movements of the crusher relative to the falling material.

Expanded, the dependency for defining the material path will be as follows:

$$
I_{1}=\frac{g\left(\sin [\alpha]-\mu_{f} \cos [\alpha]\right)}{4 f}+\frac{F}{m_{c r} \omega^{2}},
$$

where $\mu_{f}$-coefficient of friction; $m_{c r}$ - the mass of the vibration parts of the machine (mass of the crusher].

Knowing the total length of the crushing plate, let's find the number of cycles of application of loading to the material during its stay in the crushing chamber $\left(h / l_{1}\right)=n_{i}$.

On the other hand, the required number of load cycles for material destruction is calculated by the formula [2]:

$$
n_{i}^{\prime}=\left(\delta_{d} / \delta_{e}\right)
$$

where $\delta_{d}$ - depth of entry of the crushing plate into the body of the crushed material, at which the material is destroyed, $\mathrm{m} ; \delta_{e}$ - depth of entry of the crushing plate into the body of the material at a given energy of a single impact.

The value $\delta_{e}$ depends on the dependence [2]:

$$
\delta_{e}=(2, v) /\left(c_{w} k_{d} S_{c}\right)
$$

where $E_{i}=m v^{2} / 2$ - impact energy, $\mathrm{J} ; v$ - speed of application of the load to the material, $\mathrm{m} / \mathrm{s}$; $C_{w}$ - wave propagation speed, $\mathrm{m} / \mathrm{s} ; k_{d}-$ dynamic factor; $S_{c}$ - contact area, $\mathrm{m}^{2}$.

\subsection{INVESTIGATION OF THE STRESS-STRAIN STATE OF THE MATERIAL}

\section{Study of the stress-strain state of a solid material.}

The stresses arising on the platforms of the selected element are shown in Fig. 2.2. 


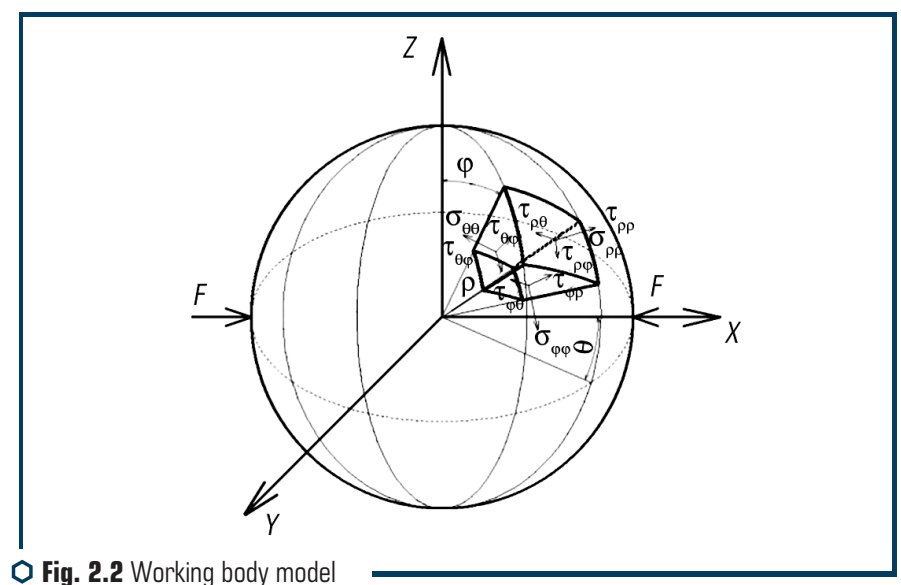

A complete description of the stress-strain state in the linear theory of elasticity (spherical coordinates] includes the following dependences [3]:

- static equations:

$\left\{\begin{array}{l}\frac{\partial \sigma_{\rho \rho}}{\partial \rho}+\frac{1}{\rho \sin (\varphi)} \frac{\partial \tau_{\rho \theta}}{\partial \theta}+\frac{1}{\rho} \frac{\partial \tau_{\rho \varphi}}{\partial \varphi}+\frac{1}{\rho}\left(2 \sigma_{\rho \rho}-\sigma_{\theta \theta}-\sigma_{\varphi \varphi}+\tau_{\rho \varphi} \operatorname{ctg}(\varphi)\right)+M=0 ; \\ \frac{\partial \tau_{\rho \theta}}{\partial \rho}+\frac{1}{\rho \sin (\varphi)} \frac{\partial \sigma_{\theta \theta}}{\partial \theta}+\frac{1}{\rho} \frac{\partial \tau_{\theta \varphi}}{\partial \varphi}+\frac{1}{\rho}\left(\tau_{\theta \varphi}(3+2 \operatorname{ctg}(\varphi))\right)+N ; \\ \frac{\partial \tau_{\varphi \rho}}{\partial \rho}+\frac{1}{\rho \sin (\varphi)} \frac{\partial \tau_{\theta \varphi}}{\partial \theta}+\frac{1}{\rho} \frac{\partial \sigma_{\varphi \varphi}}{\partial \varphi}+\frac{1}{\rho}\left(\left(\sigma_{\rho \rho}-\sigma_{\theta \theta}\right) \operatorname{ctg}(\varphi)-3 \tau_{\varphi \rho}\right)+L=0 ;\end{array}\right.$

- deformation equations:

$$
\left\{\begin{array}{l}
\varepsilon_{\rho \rho}=\frac{\partial u}{\partial \rho} ; \varepsilon_{\varphi \varphi}=\frac{1}{\rho} \frac{\partial w}{\partial \varphi}+\frac{u}{\rho} ; \varepsilon_{\theta \theta}=\frac{1}{\rho}\left(\frac{1}{\sin (\varphi)} \frac{\partial v}{\partial \theta}+u+w \operatorname{ctg}(\varphi)\right) \\
\gamma_{\rho \theta}=\frac{1}{\rho} \frac{1}{\sin (\varphi)} \frac{\partial u}{\partial \theta}+\rho \frac{\partial}{\partial \rho}\left(\frac{v}{\rho}\right) ; \gamma_{\rho \varphi}=\rho \frac{\partial}{\partial \rho}\left(\frac{w}{\rho}\right)+\frac{1}{\rho} \frac{\partial u}{\partial \varphi} ; \\
\gamma_{\theta \varphi}=\frac{1}{\rho} \frac{1}{\sin (\varphi)}\left(\frac{\partial}{\partial \varphi}(v \sin (\varphi))+\frac{\partial w}{\partial \theta}\right)
\end{array}\right.
$$

- stress equations:

$$
\left\{\begin{array}{l}
\sigma_{\rho \rho}=2 G \varepsilon_{\rho \rho}+\lambda e ; \tau_{\rho \theta}=G \gamma_{\rho \theta} ; \\
\sigma_{\theta \theta}=2 G \varepsilon_{\theta \theta}+\lambda e ; \tau_{\rho \varphi}=G \gamma_{\rho \varphi} ; \\
\sigma_{\varphi \varphi}=2 G \varepsilon_{\varphi \varphi}+\lambda e ; \tau_{\theta \varphi}=G \gamma_{\theta \varphi},
\end{array}\right.
$$


where $\rho, \theta, \varphi$ - suitable coordinates for the placement of the stressed body element; $u, v, w$ - movement in the direction of the corresponding coordinate axes; $\sigma_{\rho \rho}, \sigma_{\theta \theta}, \sigma_{\varphi \varphi}-$ normal stresses at the respective sites; $\tau_{\rho \theta}, \tau_{\rho \varphi}, \tau_{\theta \varphi}$ - shear stresses at the respective sites; $M, N, L$-volumetric forces; $G=E / 2(1+v)-$ shear modulus; $v=\lambda / 2(\lambda+G)-$ Poisson's ratio; $\lambda=E v /((1+v)(1-2 v))-$ Lame constant; $e=1 / \rho^{2} \partial\left(\rho^{2} u\right) / \partial \rho+(1 / \rho \sin (\varphi))((\partial(w \sin (\varphi)) / \partial \varphi)+\partial v / \partial \theta)-$ volumetric relative deformation; $\varepsilon_{\rho \rho}, \varepsilon_{\theta \theta}, \varepsilon_{f f}$ - linear deformations; $\gamma_{\rho \theta}, \gamma_{\rho f}, \gamma_{\theta f}$-shear deformation.

To solve the problem, let's use the displacement method.

Combining equations (2.15)-(2.17) by alternate substitution, and performing some mathematical transformations, let's obtain the equation of elastic balance of the body in displacements [4]:

$$
\left\{\begin{array}{l}
(\lambda+2 G) \rho \sin (\varphi) \partial e / \partial \rho-2 G\left[\partial \xi_{\varphi} / \partial \theta-\partial\left(\xi_{\theta} \sin (\varphi)\right) / \partial \varphi\right]=0 \\
(\lambda+2 G)(1 / \sin (\varphi)) \partial e / \partial \theta-2 G\left[\partial \xi_{\rho} / \partial \varphi-\partial\left(\rho \xi_{\varphi}\right) / \partial \rho\right]=0 ; \\
(\lambda+2 G) \sin (\varphi) \partial e / \partial \varphi-2 G\left[\partial\left(\rho \xi_{\theta} \sin (\varphi)\right) / \partial \rho-\partial \xi_{\rho} / \partial \theta\right]
\end{array}\right.
$$

where $\xi_{\rho}, \xi_{\theta}, \xi_{f}$ - components of rotation, determined by the following formulas:

$$
\left\{\begin{array}{l}
\xi_{\rho}=1 / 2 \rho \sin (\varphi)[\partial w / \partial \theta-\partial(v \sin (\varphi)) / \partial \varphi] \\
\xi_{\theta}=1 / 2 \rho[\partial u / \partial \varphi-\partial(\rho w) / \partial \rho] \\
\xi_{\varphi}=1 / 2 \rho \sin (\varphi)[\partial(\rho v \sin (\varphi)) / \partial \rho-\partial u / \partial \theta] .
\end{array}\right.
$$

Therefore, to solve this problem, it is necessary to find the displacement functions. These functions must satisfy three main conditions [4]:

1) be harmonious or biharmonic functions;

2) transform equation (2.18) into an identity;

3) stresses, expressed in terms of displacement functions, must satisfy the compatibility equations on the surface, which are written as follows [5]:

$$
\left\{\begin{array}{l}
\sigma_{\rho v}=\sigma_{\rho \rho} l+\tau_{\rho \theta} m+\tau_{\rho \varphi} n ; \\
\sigma_{\theta v}=\tau_{\theta \rho} l+\sigma_{\theta \theta} m+\tau_{\varphi \rho} n ; \\
\sigma_{\varphi v}=\tau_{\varphi \rho} l+\tau_{\varphi \theta} m+\sigma_{\varphi \varphi} n,
\end{array}\right.
$$

where $I, m, n$ - direction cosines.

In expanded form, the displacement functions for a spherical coordinate system are written as follows [6]:

$$
\left\{\begin{array}{l}
2 G u_{1}=4(1-v)\left[\left(\chi_{1} \cos \theta+\chi_{2} \sin \theta\right) \sin \varphi+\chi_{3} \cos \varphi\right]-\partial M / \partial \rho \\
2 G w_{1}=4(1-v)\left[\left(\chi_{1} \cos \theta+\chi_{2} \sin \theta\right) \cos \varphi-\chi_{3} \sin \varphi\right]-(1 / \rho)(\partial M / \partial \varphi) \\
2 G v_{1}=4(1-v)\left[\left(-\chi_{1} \sin \theta+\chi_{2} \cos \theta\right)\right]-(1 / \rho \sin \varphi)(\partial M / \partial \theta)
\end{array}\right.
$$

where $M=\chi_{0}+\rho\left[\left(\chi_{1} \cos \theta+\chi_{2} \sin \theta\right) \sin \varphi+\chi_{3} \cos \varphi\right]$. 
In this problem, the body forces are insignificant, so they can be neglected.

Therefore, from the above, it is possible to conclude that the solution of equations (2.21) is reduced to finding three harmonic functions $\chi_{1}, \chi_{2}, \chi_{3}$.

Such functions are volumetric spherical functions, which, in general, can be written as follows:

$$
\chi=\sum\left(A_{n} \rho^{n}+B_{n} \rho^{-n-1}\right)\left(C_{m} \cos (m \theta)+D_{m} \sin (m \theta)\right)\left(L_{n m} P_{n m}(\mu)+F_{n m} Q_{n m}(\mu)\right),
$$

where $\mu=\cos [\varphi]$ - module; $P_{n m}(\mu)$ - Legendre function of the first kind, degree $n$ and order $m$; $Q_{n m}(\mu)$ - Legendre function of the second kind, degree $n$ and order $m ; Q_{n}(\mu)$ - Legendre function of the second kind; $P_{n}(\mu)$ - Legendre function of the first kind; $A_{n}, B_{n}, C_{m}, D_{m}, L_{n, m}, F_{n, m}-$ constants.

Now, knowing the displacement by formulas (2.16), (2.17), it is possible to find deformations and stresses. Let's consider the most atypical condition under which $n 1-n 3, m 1-m 3$ are different integers, for this $-1<\mu<1$. For subsequent calculations, let's take: $n 1=4, m 1=1 ; n 2=5$, $m 2=2 ; n 3=6, m 3=3$. Then equations (2.22) can be written as follows:

$$
\begin{aligned}
& \chi_{1(m 1,11)}=-\left(\left(\rho^{9} A_{4}+B_{4}\right)\left(\sin [\theta] H_{1}+\cos [\theta] M_{1}\right) / 12 \sqrt{1-\mu^{2}} \rho^{5}\right) \times \\
& \times\left(\left(\begin{array}{l}
-32+230 \mu^{2}-210 \mu^{4}+ \\
+15 \mu\left(3-10 \mu^{2}+7 \mu^{4}\right) \log \left[\frac{1+\mu}{1-\mu}\right]
\end{array}\right) F_{4,1}+30 \mu\left(3-10 \mu^{2}+7 \mu^{4}\right) L_{4,1}\right) \text {; } \\
& \chi_{2(m 2, n 2)}=-\frac{\left(\rho^{11} A_{5}+B_{5}\right)\left(\sin [2 \theta] H_{2}+\cos [2 \theta] M_{2}\right)}{4\left(-1+\mu^{2}\right) \rho^{6}} \times \\
& \times\left(\begin{array}{l}
\left.\left(64-686 \mu^{2}+1260 \mu^{4}-630 \mu^{6}+105 \mu\left(-1+\mu^{2}\right)^{2}\left(-1+3 \mu^{2}\right) \log \left[\frac{1+\mu}{1-\mu}\right]\right) F_{5,2}+\right) \\
+210 \mu\left(-1+\mu^{2}\right)^{2}\left(-1+3 \mu^{2}\right) L_{5,2}
\end{array}\right) \\
& \chi_{3(m 3, n 3)}=-\left(\left(\rho^{13} A_{6}+B_{6}\right)\left(\sin [3 \theta] H_{3}+\cos [3 \theta] M_{3}\right) / 4\left(1-\mu^{2}\right)^{3 / 2} \rho^{7}\right) \times \\
& \left.\times\left(\begin{array}{l}
-512+7326 \mu^{2}-20286 \mu^{4}+ \\
+20370 \mu^{6}-6930 \mu^{8}+315 \mu\left(-1+\mu^{2}\right)^{3} \times \\
\times(-3+11 \mu)^{2} \log \left[\frac{1+\mu}{1-\mu}\right]
\end{array}\right) F_{6,3}+630 \mu\left(-1+\mu^{2}\right)^{3}\left(-3+11 \mu^{2}\right) L_{6,3}\right) .
\end{aligned}
$$
of destruction.

The realistic crack shape is an elliptical shape. In this form, by changing the ellipticity, it is possible to realize different degrees of curvature of the crack boundary.

The solution to this problem is complex and very voluminous, due to the use of biharmonic functions. Therefore, for further research, let's use the technique proposed for solving the stressstrain state of a body near the rupture zone, which has the shape of an ellipse [3, 4]. 
In general, the problem is divided into two separate ones:

1) an elliptical crack under internal pressure;

2) an elliptical crack under shear conditions.

For a better understanding of the problem statement, let's present a diagram of the location of a plane crack in a three-dimensional body (Fig. 2.3).

The limiting conditions for solving the problem of an elliptical crack under the action of loading are as follows:

$$
\sigma_{z}=-F, \quad \xi=0, w=0, \varrho=0 .
$$

To solve the problem, the gravitational potential outside a homogeneous elliptical plate of the following form was used [4]:

$$
\varphi=\frac{-}{2} \int_{\xi}^{\infty}\left[\frac{x^{2}}{a^{2}+\int}+\frac{y^{2}}{b^{2}+\int}+\frac{z^{2}}{\int}-1\right] \frac{d \int}{V\left(\int\right)}
$$

where $V\left(\int\right)=\left[\int\left(a^{2}+\int\right)\left(b^{2}+\int\right)\right]^{1 / 2}-$ function of $\int ; \int$ - continuous integration; $a, b$ - respectively large and having semi-axes of an ellipse.

Constant $A$ is found by the following formula [4]:

$$
A=-a b^{2} F / 4 \mu E(m)
$$

where $E(m)=\int_{0}^{\pi / 2} \sqrt{1-k^{2}(\sin [\varphi])^{2}} d \varphi$ - complete elliptic integral of the second kind; $\varphi$ - amplitude of the integral; $m=\sin [\alpha]$ - modulus of the integral; $\alpha$ - modular angle.

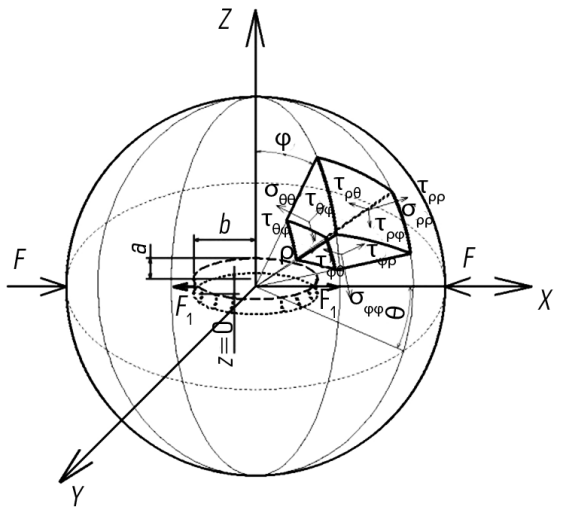

O Fig. 2.3 Scheme for determining the stress-strain state in the presence of a rupture in the crushing material 
After some transformations and calculations, the displacements from the action of normal forces through the elliptical coordinates will have the following form [4]:

$$
\begin{aligned}
& u_{n}=\frac{2 A x}{a b^{2}}\left\{(1-2 v)\left[u_{f}+E\left[u_{f}\right]\left(\frac{m^{\prime}}{m}\right)^{2}\right]-\frac{b z\left[\left(b^{2}+\xi\right) \varrho \vartheta\right]^{1 / 2}}{(\xi-\rangle)(\xi-\vartheta)\left(a^{2}+\xi\right)^{1 / 2}}\right\}, \\
& v_{n}=\frac{2 A y}{a b^{2}}\left\{(1-2 v)\left[E\left[u_{f}\right]-m^{\prime 2} u_{f}-m^{2} \frac{s n u_{f} \cdot c n u_{f}}{d n u_{f}}\right] \frac{1}{m^{2}}-\frac{b z\left[\left(a^{2}+\xi\right) \varrho \vartheta\right]^{1 / 2}}{(\xi-\varrho)(\xi-\vartheta)\left(b^{2}+\xi\right)^{1 / 2}}\right\}, \\
& w_{n}=\frac{2 A z}{a b^{2}}\left\{\begin{array}{l}
(1-2 v) E\left[u_{f}\right]+\left[\frac{c n u_{f}}{d n u_{f}}-2(1-v) \frac{d n u_{f}}{c n u_{f}}\right] s n u_{f}+ \\
\left.+\frac{\xi^{1 / 2}\left[\xi\left(a^{2} b^{2}-\varrho \vartheta\right)-a^{2} b^{2}(\varrho+\vartheta)-\left(a^{2}+b^{2}\right) \varrho \vartheta\right]}{a(\xi-\varrho)(\xi-\vartheta)\left(a^{2}+\xi\right)^{1 / 2}\left(b^{2}+\xi\right)^{1 / 2}}\right]
\end{array}\right.
\end{aligned}
$$

where $\xi, \varrho, \vartheta$ - elliptical coordinates; $\xi=$ const - family of ellipsoids; $\varrho$ - hyperboloid with one cavity; $\vartheta$ - hyperboloids with two cavities; $E\left[u_{f}\right]=\int_{0}^{u_{f}} d n^{2} \beta d \beta$ - function; $u_{f}$-variable; $m^{\prime}-$ argument of elliptic functions (modulus of the integral), $\left(m^{\prime}\right)^{2}+(m)^{2}=1 ; s n u_{f}, c n u_{f}, d n u_{f}$ - elliptic functions of C. Jacobi.

Now let's return to solving the general problem of a body with a crack. Using the principle of superposition of the stress states of two problems, namely, the stress-strain state of a body without a crack and in the vicinity of a crack, it is possible to write the following equations:

$$
u_{t o t}=u_{i}+u_{n i} ; \sigma_{t o t}=\sigma_{i}+\sigma_{n i},
$$

where $u_{i}, \sigma_{i}$ - displacement and stress, respectively, calculated for a body without a crack; $u_{n i}, \sigma_{n i}-$ corresponding displacements and stresses calculated for bodies with a rupture zone.

Since the displacements indicated in formulas (2.27) were determined for elliptical coordinates, and preliminary calculations were performed for a spherical system, therefore, when performing further calculations, it is necessary to switch to a single coordinate system. Let's accept the spherical coordinate system as more convenient for further operation.

After simple transformations of the system of equations (2.27), let's obtain the value of the displacements in spherical coordinates:

$$
\begin{aligned}
& u_{n}=\frac{A}{3 r^{7}}\left(\begin{array}{l}
21 r^{5}-70 r^{4} \rho+105 r^{3} \rho^{2}- \\
-84 r^{2} \rho^{3}+35 r \rho^{4}-6 \rho^{5}
\end{array}\right)(-3+(-5+4 v) \cos [2 \varphi])+0[\rho-r]^{6} ; v_{n}=0 \\
& w_{n}=-\frac{2 A}{3 r^{7}}(-1+2 v)\left(\begin{array}{l}
21 r^{5}-70 r^{4} \rho+105 r^{3} \rho^{2}- \\
-84 r^{2} \rho^{3}+35 r \rho^{4}-6 \rho^{5}
\end{array}\right) \sin [2 \varphi]+O[\rho-r]^{6}
\end{aligned}
$$


It should be noted that these equations were obtained on the basis of the expansion of formulas (2.27) in power series. The calculations took into account the condition $-\xi \approx \rho^{2} ; \varrho \approx \vartheta \approx 0$.

To obtain the stress components, it is necessary to substitute formulas (2.29) into the equation for determining the stresses.

Finally, the stresses in the body in the presence of a rupture zone will be equal:

$$
\begin{aligned}
& \left(G\left(\begin{array}{l}
-70 r^{4}+210 r^{3} \rho-252 r^{2} \rho^{2}+ \\
+140 r \rho^{3}-30 \rho^{4}
\end{array}\right)(-3+(-5+4 v) \cos [2 \varphi])-\right. \\
& \sigma_{\rho \rho}=\frac{1}{3 r^{7}} 2 A\left(-1 / \rho \lambda\left(\begin{array}{l}
42 r^{5}(1+v)-35 r^{4}(7+4 v) \rho+105 r^{3}(5+2 v) \rho^{2}- \\
-42 r^{2}(13+4 v) \rho^{3}+70 r(4+v) \rho^{4}-3(19+4 v) \rho^{5}+ \\
+3\left(\begin{array}{l}
14 r^{5}(1+v)-105 r^{4} \rho-35 r^{3}(-7+2 v) \rho^{2}+ \\
+14 r^{2}(-19+8 v) \rho^{3}-70 r(-2+v) \rho^{4}+ \\
+(-29+16 v) \rho^{5}
\end{array}\right) \cos [2 \varphi]
\end{array}\right)\right) ;
\end{aligned}
$$

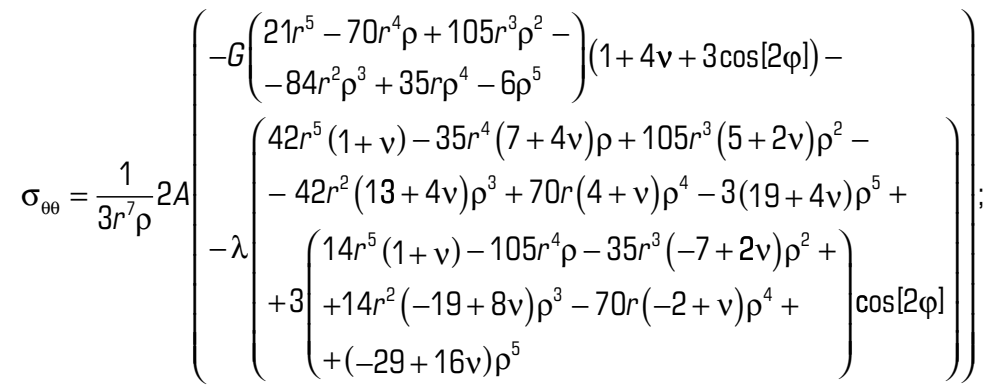

$$
\begin{aligned}
& \sigma_{\varphi \varphi}=-\frac{1}{3 r^{7} \rho} 2 A\left(\begin{array}{l}
3 G\left(21 r^{5}-70 r^{4} \rho+105 r^{3} \rho^{2}-84 r^{2} \rho^{3}+35 r \rho^{4}-6 \rho^{5}\right)+ \\
+\lambda\left(\begin{array}{l}
42 r^{5}(1+v)-35 r^{4}(7+4 v) \rho+105 r^{3}(5+2 v) \rho^{2}- \\
-42 r^{2}(13+4 v) \rho^{3}+70 r(4+v) \rho^{4}-3(19+4 v) \rho^{5}
\end{array}\right)+ \\
+\left(\begin{array}{l}
G(1+4 v)\left(\begin{array}{l}
14 r^{5}(1+v)-105 r^{4} \rho-35 r^{3}(-7+2 v) \rho^{2}+ \\
-84 r^{2} \rho^{3}+35 r r^{4}-6 \rho^{5}
\end{array}\right)+105 r^{3} \rho^{2}- \\
+14 r^{2}(-19+8 v) \rho^{3}-70 r(-2+v) \rho^{4}+ \\
+(-29+16 v) \rho^{5}
\end{array}\right)
\end{array}\right) \\
& \tau_{\rho \theta}=0 ; \tau_{\rho \varphi}=-\frac{4 A G}{3 r^{7} \rho}\left(\begin{array}{l}
21 r^{5}(-2+v)-35 r^{4}(-5+4 v) \rho+ \\
+315 r^{3}(-1+v) \rho^{2}-42 r^{2}(-7+8 v) \rho^{3}+ \\
+35 r(-4+5 v) \rho^{4}+9(3-4 v) \rho^{5}
\end{array}\right) \sin [2 \varphi] ; \tau_{\theta \varphi}=0 \text {. }
\end{aligned}
$$


Thus, knowing all the components of stresses and displacements for two stress-strain states, it is possible to determine the total energy of destruction.

Based on the superposition of equations for determining displacements without a crack and equations for determining the displacements of a body with a crack, the corresponding graphs were constructed [4]. The graphs of the dependence of the principal stresses on the size of the major semiaxis of the crack ellipse are shown in Fig. 2.4. The graph of stress versus zenith angle $\varphi$ is shown in Fig. 2.5. The change in stress $\sigma \rho$ depending on the radius $r$ is shown in Fig. 2.6.

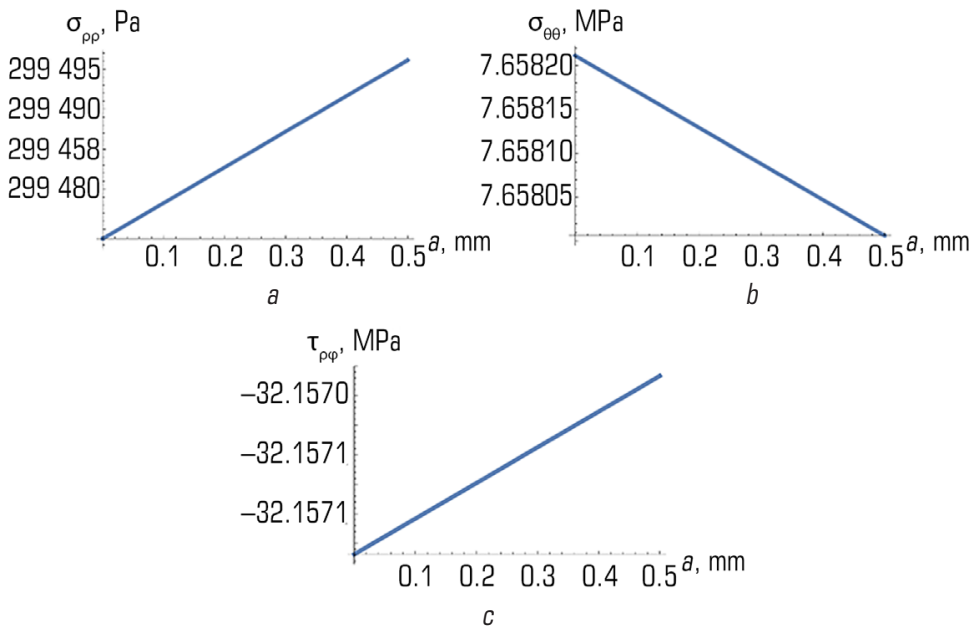

O Fig. 2.4 Graph of stress dependence on the size of the major semiaxis of the ellipse: $a-\sigma \rho-a ; b-\sigma \theta-a ; c-\tau \rho \varphi-a$

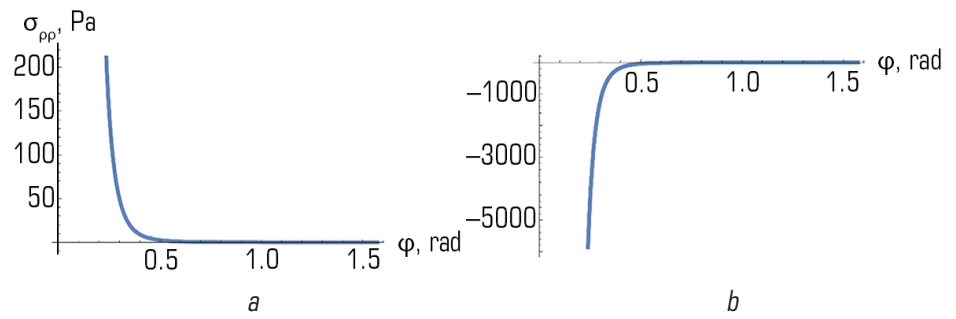

Fig. 2.5 Graph of stress changes depending on the angle of application of the force $\varphi$ : $a-\sigma \rho-\varphi ; b-\tau \rho \varphi-\varphi$ 


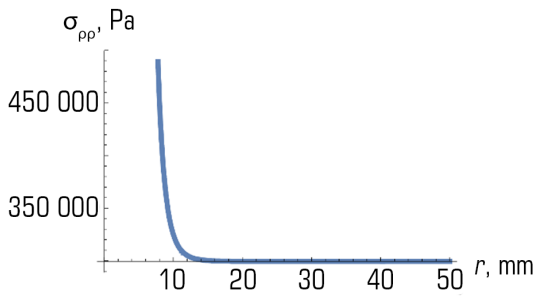

Fig. 2.6 Graph of changes in normal stresses $\sigma_{\rho}$ depending on the radius $r$

As follows from the graphs (Fig. 2.4), the dependences of stresses on the size of the major semiaxis of the ellipse have a linear characteristic within the framework of the calculations, the numerical values of which were used to determine the parameters of the crusher.

The significant influence of the angle of application of the force on the change in stresses is noted in the range of $0.3-0.5$ (Fig. 2.5).

A more complex law of variation of normal stresses depending on the radius $r$ is noted in the range of 9-12 $\mathrm{mm}$ (Fig. 2.6).

\subsection{RESEARCH AND ANALYSIS OF MATERIAL GRINDING MACHINE PARAMETERS}

A mathematical model of an experimental jaw crusher. The physical model of a vibration jaw crusher is shown in Fig. 2.7. When considering the physical model, the structural elements of the machine take an elementary form, the gravity forces of individual links are applied at certain points, the forces of resistance to destruction are presented in the form of a total force applied to the jaw center $[7,8]$.

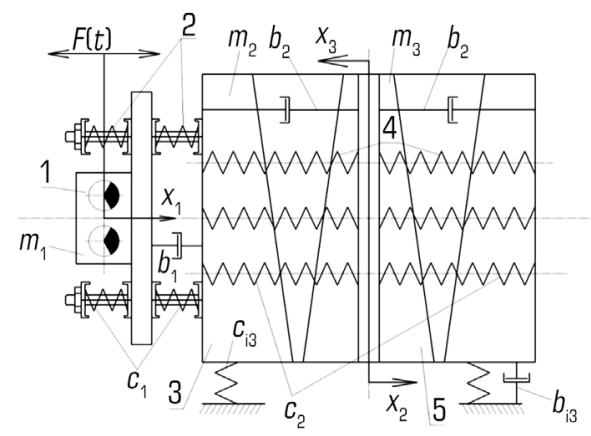

Fig. 2.7 Physical model of a three-mass vibration crusher 
When compiling the equations of motion of the vibration system of a jaw crusher, let's use Hamilton's variational principle. It establishes that the variation in kinetic and potential energy plus variation in the work of non-conservative forces during any time interval should be equal to zero [9-11].

To compose the equations of motion, let's consider the structural diagram of a vibration jaw crusher. The investigated jaw crusher is made in the form of a three-mass resonance system (Fig. 2.7), in which rectilinear vibrations are realized and the dynamics of which occurs according to a three-mass scheme.

The vibration exciter 1 with the base plate is the first or active mass, the movable body 3 is the second mass, the middle plate with inner armor 5 is the third mass. Each mass performs horizontal oscillations along the $\mathrm{x}$-axis along the generalized coordinates, respectively, and has its own inertial parameter.

The active mass is set in motion by the centrifugal force arising from the rotation of the unbalanced masses of the vibration exciter. The crusher is adjusted in such a way that during operation the first and third masses oscillate in phase, and the second in antiphase, that is, when one chamber approaches in another, the jaw diverge.

All masses of the crusher are connected in pairs by elastic systems 2 and 5 (Fig. 2.7) with stiffnesses and. The vibration machine is isolated from the foundation by vibration dampers with stiffness on which the second mass rests. Resistance forces act in the system, but these forces can be neglected to simplify calculations.

The system of equations of motion for the model using the Lagrange equation of the second kind will take the form [5]:

$$
\left\{\begin{array}{l}
m_{1} \ddot{x}_{1}+2 x_{1} c_{1}-x_{2} c_{1}=F_{0} \sin \omega t \\
\left(m_{2}+k m_{m}\right) \ddot{x}_{2}+x_{2} c_{1}-x_{1} c_{1}+2 c_{2} x_{2}-2 c_{2} x_{3}+x_{2} c_{f}=0 \\
\left(m_{3}+k m_{m}\right) \ddot{x}_{3}+2 c_{2} x_{3}-2 c_{2} x_{2}=0 \\
F_{c r} \leq c_{2} x_{n} \\
x_{1}>0 ; x_{3}>0 ; x_{2}<0 \\
x_{2}+x_{3}=\xi \cdot D_{\max }
\end{array}\right.
$$

where $F_{0}$ - centrifugal force $\left(F_{0}=m_{0} r_{0} \omega^{2}\right) ; r_{0}$ - eccentricity, distance from the center of gravity of the mass $m_{0}$ to the center of the axis of rotation, $\mathrm{m} ; \omega$-angular speed of unbalance rotation, $\mathrm{s}^{-1}$; $m_{1}, m_{2}, m_{3}$ - the first, second and third mass of the crusher, $\mathrm{kg} ; c_{1}, c_{2}$ - stiffness of the springs connecting the masses of the crusher; $x_{1}, x_{2}$ and $x_{3}$-corresponding displacement of the masses of the crusher, $\mathrm{mm} ; c_{f}$ - stiffness of the connections of the system connecting the machine to the foundation; $F_{c r}$ - crushing force formula, $\mathrm{N} ; \xi$ - relative compression of the material; $D_{\max }-$ the maximum size of the crushed material, $\mathrm{mm}$. 
The solution to the system of equations (2.30) and (2.31) is obtained in the form:

$$
\left\{\begin{array}{l}
x_{1}=X_{1} \sin \omega t \\
x_{2}=X_{2} \sin \omega t \\
x_{3}=X_{3} \sin \omega t
\end{array}\right.
$$

where $X_{1}, X_{2}, X_{3}$ - amplitudes of mass displacement.

Substituting (2.32) into (2.18) and carrying out the appropriate transformations, let's obtain the dependences for determining the values of the amplitudes of the displacement of the masses $X_{1}, X_{2}, X_{3}$.

The energy consumed by the electric motor is spent on the work on crushing the material, on the potential energy of deformation of the elastic system, on overcoming friction forces, on heat losses, on the deformation of the crusher parts and other losses. The greatest energy consumption during the operation of the crusher is spent on crushing the material, the kinetic energy of the crushing plates and the potential energy of deformation of the springs.

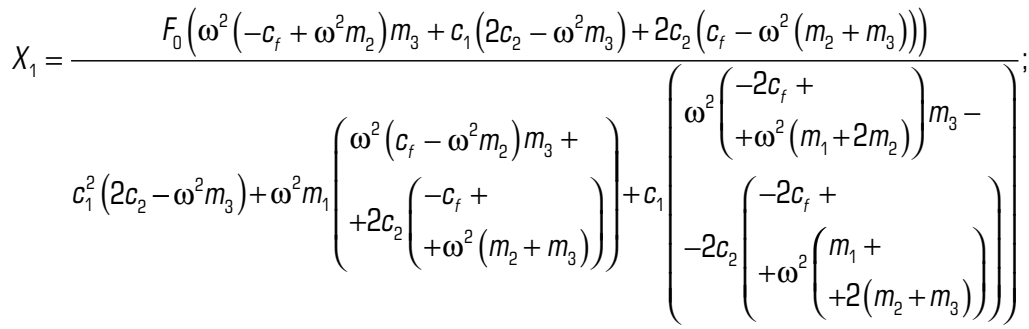

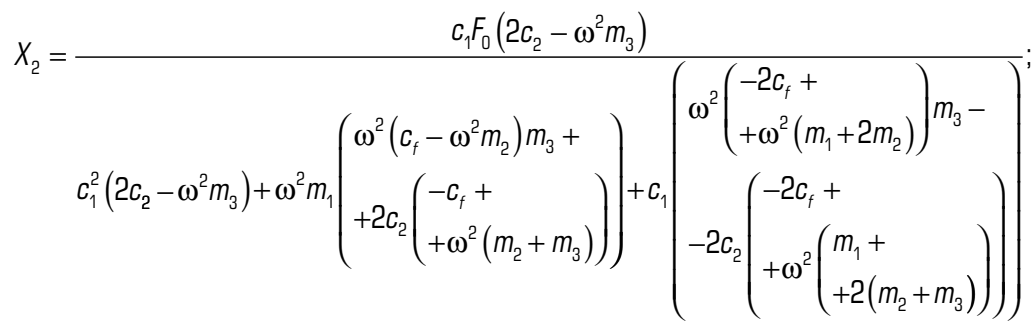

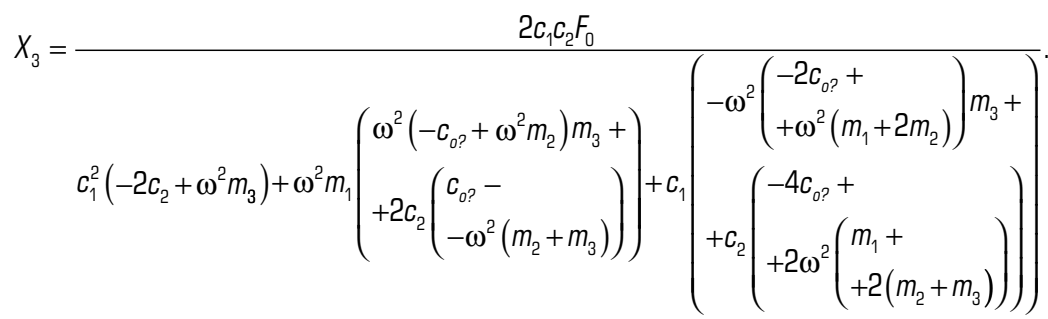


Thus, the total energy consumed by the crusher for crushing the material will look like this:

$$
E_{\text {tot }}=C+P
$$

where $C$ - kinetic energy of the system; $P$ - potential energy of the system.

Part of the potential energy is spent on the destruction of the material. Knowing the general displacements arising in the material during its destruction and applying the condition of the minimum potential energy of deformation, it is possible to write the following:

$$
U_{p}=G \iiint\left[\varepsilon_{\rho \rho}^{2}+\varepsilon_{\theta \theta}^{2}+\varepsilon_{\varphi \varphi}^{2}+\frac{v}{1-2 v} e^{2}+\frac{1}{2}\left(\gamma_{\rho \theta}^{2}+\gamma_{\theta \varphi}^{2}+\gamma_{\rho \varphi}^{2}\right)\right] \rho^{2} \sin \varphi d \rho d \theta d \varphi .
$$

Knowing the energy consumption for the destruction of the material, one can proceed to determining the energy consumption for the process as a whole.

Let's consider two vibration-driven crushing systems shown in Fig. $\mathbf{2 . 8 .}$

The vibration jaw crusher (Fig. 2.8, a) is made in the form of a three-mass resonance system, in which rectilinear vibrations are realized and the dynamics of which occurs according to a threemass scheme. The vibration exciter 1 with the base plate is the first or active mass, the movable body 5 is the second mass, the middle plate with the inner armor 4 is the third mass. All masses of the crusher are connected in pairs by elastic systems 2 and 3 .

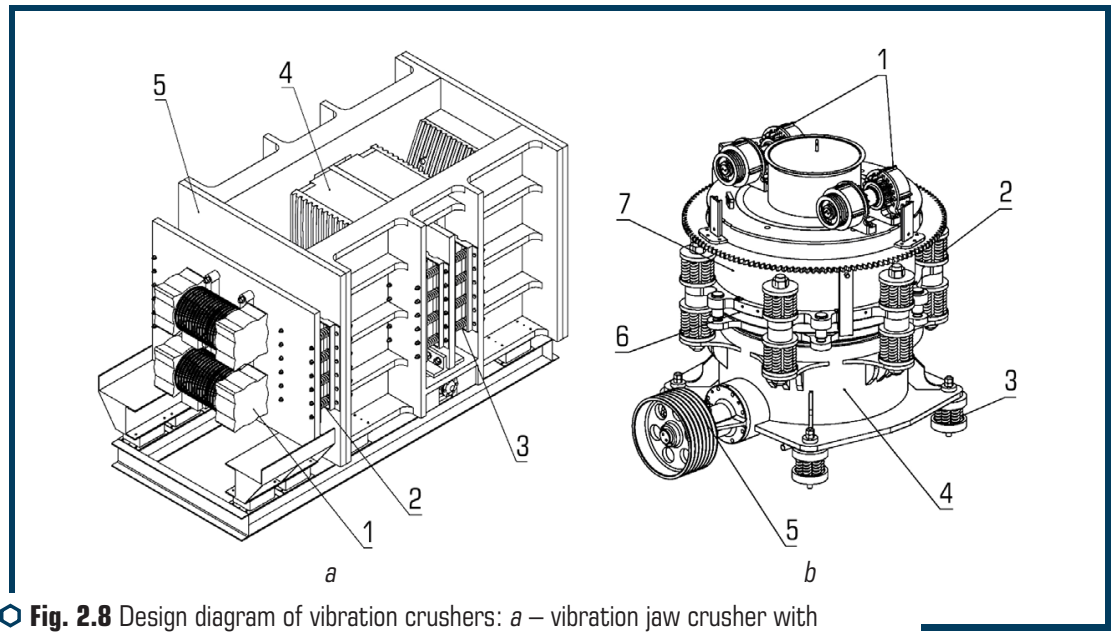
three vibration masses; $b$ - cone crusher with vibrators on the body

Vibration cone crusher (Fig. $\mathbf{2 . 8 , \boldsymbol { b }}$ ) consists of vibration exciters 1 installed on a stationary cone 7 of the crusher. The fixed cone 7 is connected to the crusher body 4 through the adjusting 
ring 6 and the elastic system 2. The crusher body 4 is installed on the foundation through the elastic system 3 . The movable crusher cone is driven through the drive 5 .

The energy consumed by the electric motors of the crushers (Fig. $\mathbf{2 . 8 ,} \boldsymbol{a}, \boldsymbol{b}$ ) is spent on the work on crushing the material. Additionally, energy costs are spent on deformation of the elastic system, overcoming friction forces, heat losses, deformation of crusher parts and other losses. However, the greatest energy consumption during the operation of crushers is spent on the kinetic energy of crushing plates and the potential energy of deformation of the springs [2].

Based on the general equation of energy consumption for a vibration jaw crusher with three vibration masses (2.32), the following equation was derived:

$$
E_{\text {tot }}=\left[\sum_{n=1}^{3} \frac{m_{n} \dot{x}_{n}^{2}}{2}\right]+\left[\frac{1}{2}\left(c_{f} x_{2}^{2}+c_{1}\left(3 x_{1}^{2}-4 x_{1} x_{2}+2 x_{2}^{2}\right)+4 c_{2}\left(x_{2}-x_{3}\right)^{2}\right)\right],
$$

where $m_{n}$ - respectively $\mathrm{n}$-mass of the crusher, $\mathrm{kg} ; c_{1}, c_{2}$ - stiffness of the springs connecting the masses of the crusher; $x_{1}, x_{2}$ and $x_{3}$ - corresponding displacements of the masses of the crusher, $\mathrm{mm}$; $c_{f}$ - the stiffness of the connections of the system connecting the machine to the foundation.

When determining the total energy of a vibration cone crusher (Fig. $\mathbf{2 . 8 , \boldsymbol { b }}$ ), let's assume that the movable cone is an absolutely rigid cylinder rolling on a solid undeformed body in the shape of an ellipse. In turn, let's assume that the crusher body with vibrators is a solid body with mass $m_{\text {tot }}$ and moment of inertia $I$, which is fixed on a fixed base by springs with stiffness $c_{i s}$ [1]:

$$
E_{\text {tot }}=\left[\frac{1}{2} m_{m c} \times\left(v_{x}^{2}+v_{y}^{2}\right)+\frac{I_{m c} \times \dot{\varphi}^{2}}{2}+\frac{1}{2} m_{s c} v_{s c}^{2}\right]+\left[\frac{c_{c r}\left(n \times x_{1}\right)^{2}}{2}+\frac{c_{1} x_{1}^{2}}{2}\right],
$$

where $T_{1}=1 / 2 m_{m c} \times\left(v_{x}^{2}+v_{y}^{2}\right)+\left(l_{m c} \times \dot{\varphi}^{2}\right) / 2$ - kinetic energy of rolling a movable cone [4]; $T_{2}=1 / 2 m_{s c} v_{s c}^{2}-$ kinetic energy of vibrations of a stationary cone; $m_{s c}$ - the mass of the movable cone; $I_{m c}$ - central moment of inertia of the cone; $x_{1}, y_{1}$ - Cartesian coordinates of the center of the cone; $\varphi$ - angle of rotation of the movable cone around its own axis; $m_{s c}$ - total mass of the stationary cone; $v_{s c}$ - speed of vertical displacements of a fixed cone; $c_{c r}$ - material stiffness coefficient; $c_{1}$ - the stiffness coefficient of the elastic system connecting the stationary cone and the crusher body.

Sandstone with a strength limit of up to $20 \mathrm{MPa}$ was taken as the model of the material of destruction. Based on the equations of motion of a vibration jaw crusher (2.31), the speeds of movement of the vibration masses of the crusher shown in Fig. 2.9.

Regarding Fig. 2.9, it should be noted that these speeds of mass fluctuations are valid at the frequency of the disturbing force $f=19 \mathrm{~Hz}$ and the total mass of the laboratory unit $m_{\text {tot }}=334 \mathrm{~kg}$.

Using the first part of equation (4) and the equations of motion of the masses of the vibration jaw crusher (2.31), graphs of the consumption of kinetic energy were built, which is spent on moving the vibration masses of the vibration crusher Fig. 2.10. 


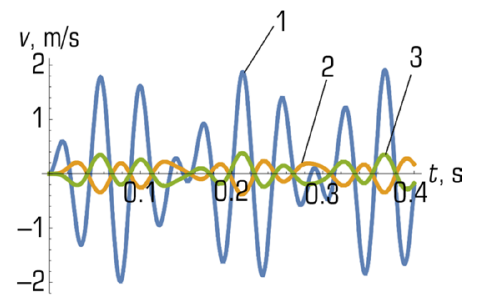

Fig. 2.9 Speeds of movement of masses of vibration jaw crusher:

1 - first mass; 2 - second mass; 3 - third mass
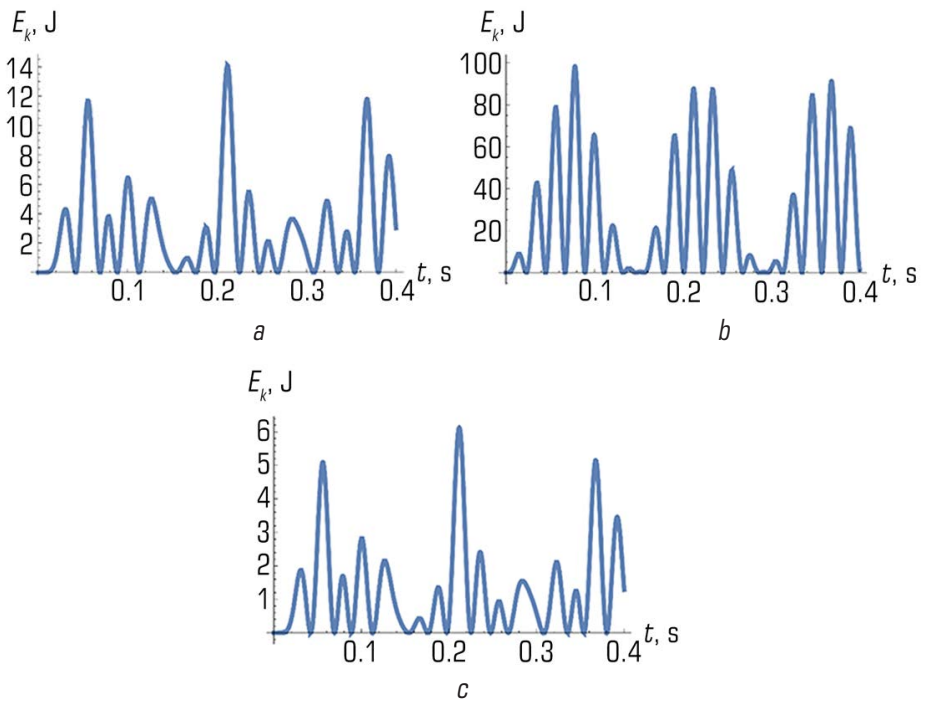

- Fig. 2.10 Graph of changes in the kinetic energy of vibration masses of a vibration jaw crusher: $a$ - first mass; $b$ - second mass; $c$ - third mass

From the graphs in Fig. 2.10, it is clear that the total kinetic energy of the motion of the masses is within $120 \mathrm{~J}$. However, equations (2.36) used to plot the graphs in Fig. 2.10 do not take into account the loss of dry and viscous friction force.

To construct graphs of potential energy consumption for the crushing process, let's use equation (2.31).

The movement of vibration masses of the crusher, determined numerically based on the equation of motion, is shown in Fig. 2.11. 


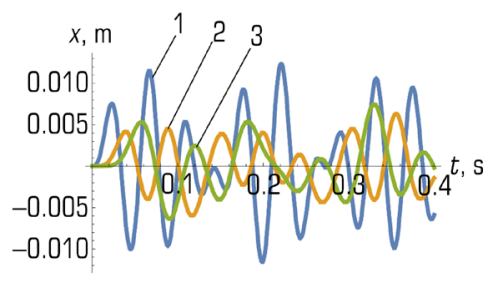

O Fig. 2.11 Change in the amplitude of vibration masses of a vibration jaw crusher: 1 - first mass; 2 - second mass; 3 - third mass

From the graph in Fig. 2.11 it can be seen that the system setting is correct, that is, the second and third masses oscillate in antiphase. The total amplitude of displacement of the second and third vibration masses is in the range of $8-10 \mathrm{~mm}$. The minimum required value of the amplitude of vibration of the crushing plates of a vibration crusher, at which destruction occurs, is within $3-5 \mathrm{~mm}$.

The graph of the total costs of potential energy for the destruction process in the crushing chamber of a vibration jaw crusher is shown in Fig. 2.11. This graph was constructed by numerous methods using equations (2.31) and (2.36).

The graphs in Fig. $\mathbf{2 . 1 0}$ and Fig. 2.12 indicate an insignificant expenditure of energy for the destruction process. It should be noted here that the system is tuned for resonant operation. That is, in the graphs in Fig. 2.10 and Fig. 2.12 does not reflect the energy consumption for the system to enter the resonance mode and maintain its operation in this mode. In addition, it should be borne in mind that, in order to simplify, the equation of motion (2.31) does not take into account energy dissipation.

To assess the energy of destruction of a body containing a crack, graphs of the instantaneous force arising when the crushing plates of the second and third masses of the vibration crusher collide with the material in Fig. 2.13. To construct this graph, equations (2.31) and (2.36) were used.

The given graph (Fig. 2.13) testifies to the adequacy of the calculation scheme to the accepted premises. So, the first mass moves in antiphase with the second mass. The third mass, accumulating energy, is an auxiliary source of energy transfer to the material crushing process.

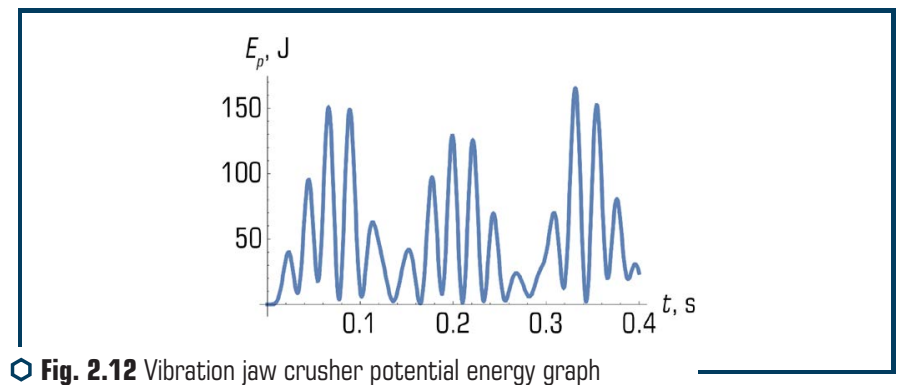




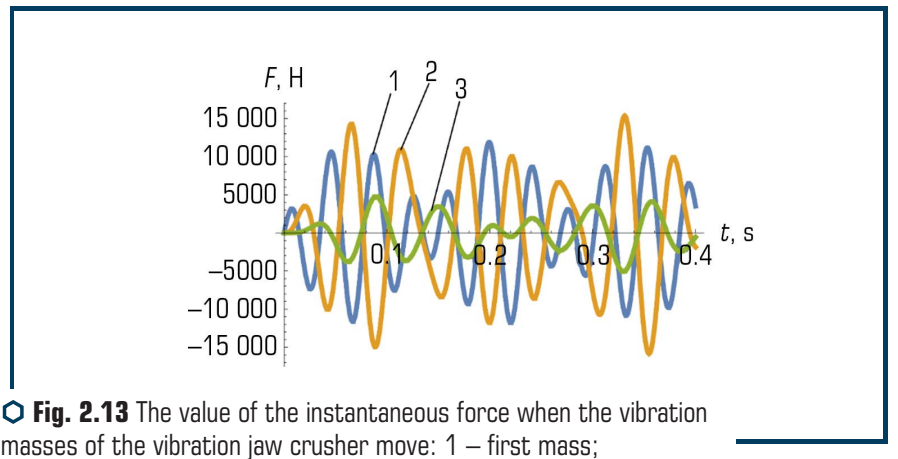

masses of the vibration jaw crusher move: 1 - first mass;

2 - second mass; 3 - third mass

In equation (2.36), to determine the energy consumption of a vibration jaw crusher, the fracture resistance of the material is considered as the added mass. That is, the indicator of the stiffness of the material is the parameter that includes energy consumption at the micro level. In equation (2.37) for a vibration cone crusher, this parameter is directly the stiffness coefficient of the material. Thus, the obtained analytical dependences (2.36), (2.37) reveal the physical essence of the grinding process, take into account the conditions of interaction between the crusher and the material to be crushed. The practical application of the obtained dependences requires the numerical values of the characteristics of a particular material, which is the subject of further research.

\subsection{DETERMINING THE OPTIMAL CHARACTERISTICS OF MATERIAL GRINDING PROCESSES}

Study of the influence of the stiffness index on the amplitude. When designing vibration machines, two modes are recognized as expedient: resonant (near resonance), with a frequency ratio $\omega / \omega_{0}=0.85 \ldots 0.95$; overresonant (only for centrifugal vibration exciters) with a significant distance from resonance, when $\omega / \omega_{0}>7[12,13]$.

The advantage of the resonant mode is to achieve a given amplitude with less forced force. The resonant mode is characterized by the stability of the amplitude in case of random changes in resistance and frequencies of natural and forced oscillations.

The operation of a vibration crusher in one of the listed modes is achieved by choosing elastic links that provide a vibration frequency, which may differ from the frequency of the forcing vibrations. The stiffness of the system depends on two main parameters of mass and vibration frequency. By manipulating these parameters, it is possible to determine the optimal stiffness value for the corresponding mode.

To begin with, for given values of the masses of the system, let's change the value of the frequency. It should be noted that the stiffness of the system should help to ensure an effective 
grinding regime for the material. To determine the stiffness of the system, let's use the following relationship [5]:

$$
c_{i j}=\left(\omega_{0} n\right)^{2} \frac{m_{i} m_{j}}{m_{i}+m_{j}}
$$

where $\omega$ - natural angular frequency of oscillations (resonant frequency); $n$ - coefficient of stiffness regulation in frequency. Substituting the corresponding values of the stiffness calculated by the formula (2.38) into the equation of the amplitudes of the displacement of masses (2.37), let's obtain the graphs of the dependence of the amplitude on the coefficient $n$ (Fig. 2.14) for the corresponding frequency modes $[6,8]$.

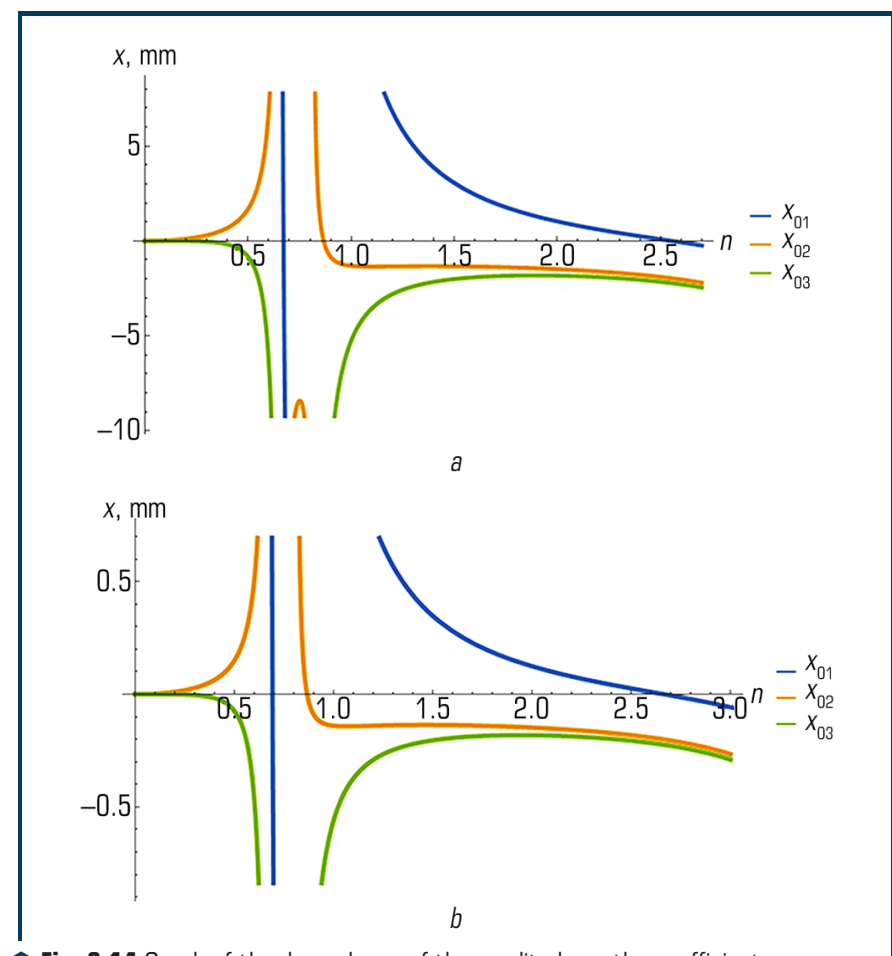

O Fig. 2.14 Graph of the dependence of the amplitude on the coefficient $n$ at the following frequency modes: $a-\omega_{0}=104.67 \mathrm{rad} / \mathrm{s} ; b-\omega \cdot \omega_{0}=314 \mathrm{rad} / \mathrm{s}$

The following conclusion can be drawn from the graphs in Fig. 2.14. The rational value of the coefficient $n$ is within $0.6 \leq n \leq 1$. With an increase in the value of the coefficient $(1 \leq n<2.5)$, the effective range of the crusher narrows and shifts towards higher frequencies. Graphs Fig. 2.14 
show that the stiffness of the elastic bonds of the crusher ensures its efficient operation in the resonance and near-resonance modes, namely, between the second and third resonances. With the value of the coefficient $0.6 \leq n \leq 1$, the amplitudes of the masses have the greatest values and decrease as $n$ approaches 1.4. With an increase in $n>1$, the change in the amplitude of the second mass is insignificant, and for $n>1.4$, the change in the amplitudes of the first and third masses is insignificant. The amplitude of the first mass reaches zero in the vicinity of the point $n=2.52$.

Study of the effect of mass on the amplitude. Rationally selected ratios of vibration masses provide the required operating mode of the crusher (in-phase, anti-phase) and provide the required vibration amplitudes of these masses [13].

When choosing the ratio of mass $m_{2}$ and $m_{2}$, the value of the mass $m_{1}$ is taken constructively, the value of the stiffness of the systems $c_{1}, c_{2}$ is taken taking into account formulas (2.31), (2.38). To introduce the mass ratio coefficient depending on the equations of motion (2.37), let's use the following logical transformations [7]:

$$
N_{23}=m_{2}+m_{3} ; k_{23}=m_{2} / m_{3} \text {. }
$$

Based on this:

$$
m_{2}=\left(N_{23} k_{23}\right) /\left(1+k_{23}\right) ; m_{3}=N_{23} /\left(1+k_{23}\right) .
$$

On the basis of the above material, the graphs of the dependence of the amplitude on the mass ratio for different frequency spectra were plotted in Fig. 2.15. Analyzing the graphs in Fig. 2.15 the following can be noted. The effective value of the coefficient $k_{23}$ for the resonant angular frequency $\omega_{0}=104.667 \mathrm{rad} / \mathrm{s}$ is within the limits $0<k_{23} \leq 2.5$, for $\omega_{0}=314 \mathrm{rad} / \mathrm{s}-$ $1.5<k_{23} \leq 2.45[14-18]$.

Analyzing each graph separately, Fig. 2.15, the following conclusions were made. At the resonant frequency $\omega_{0}=104.667 \mathrm{rad} / \mathrm{s}$, the maximum value of the amplitude of the third mass is reached in the vicinity of the point $k_{23}=1.3$. And the maximum values of the amplitudes of the first and second masses are in the vicinity of the point $k_{23}=0.75$. For the resonant frequency $\omega_{0}=314 \mathrm{rad} / \mathrm{s}$, the maximum values of the amplitudes of all three masses are achieved under the condition of the point $k_{23}$ lying in the vicinity $k_{23}=1.5$.

Let's use the same approach to find the optimal ratio of masses $m_{2}$ and $m_{1}-k_{21}$.

Construction of the amplitude-frequency characteristics of the vibration jaw crusher. Knowing all the unknown parameters of the equations of motion, graphs of the dependence of the amplitude of displacement of the crushing plates on the frequency of change in the disturbing force were built (Fig. 2.16, 2.17) [3, 5].

Based on the graphs (Fig. 2.16, 2.17), the following conclusions were made:

- the effective mode of operation of the crusher is realized in the zone of the second resonance and in the range between the second and third resonances; 
- with an increase in the calculated value of the resonant frequency of wheel vibrations $\omega_{0}$ (2.38), the range of effective operation of a three-mass vibration jaw crusher shifts towards an increase in frequency;

- the effective ranges of the crusher operation at the system stiffness values calculated at the corresponding resonant frequencies of natural vibrations $\omega_{0}$ (2.38) will be as follows:

$$
\begin{aligned}
& 100 \mathrm{rad} / \mathrm{s}<\omega<180 \mathrm{rad} / \mathrm{s} ; \omega_{0}=104.667 \mathrm{rad} / \mathrm{s} ; \\
& 97 \mathrm{rad} / \mathrm{s}<\omega<121 \mathrm{rad} / \mathrm{s} ; 186 \mathrm{rad} / \mathrm{s}<\omega<234 \mathrm{rad} / \mathrm{s} ; \\
& \omega_{0}=314 \mathrm{rad} / \mathrm{s} ; 291 \mathrm{rad} / \mathrm{s}<\omega<322 \mathrm{rad} / \mathrm{s} ; 365 \mathrm{rad} / \mathrm{s}<\omega<400 \mathrm{rad} / \mathrm{s} .
\end{aligned}
$$

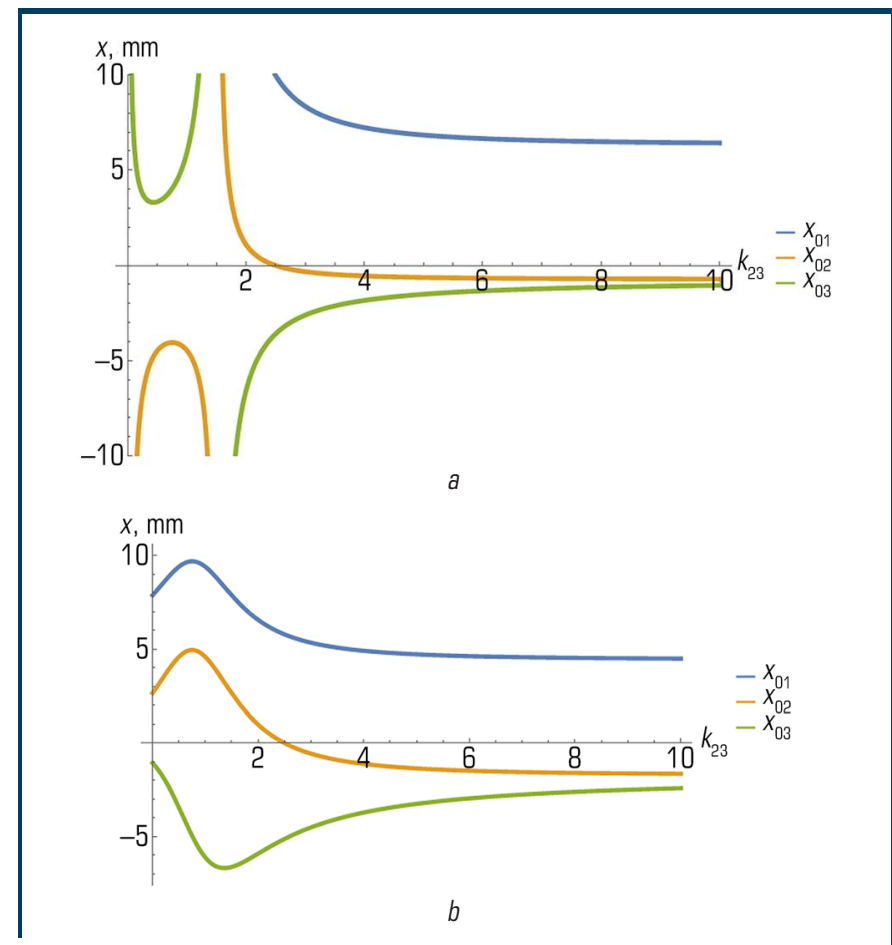

Fig. 2.15 Graphs of the dependences of the amplitude change on the mass ratio $k_{23}$ at different frequency ranges: $a-\omega_{0}=104.67 \mathrm{rad} / \mathrm{s} ; b-\omega_{0}=314 \mathrm{rad} / \mathrm{s}$

To ensure the operation of the crusher at the values of the stiffness of the system, calculated from the resonant frequency of natural vibrations of the $\omega_{0}=104.67 \mathrm{rad} / \mathrm{s}$ (2.38), in the lower 
frequency ranges, it is necessary to increase the masses of the vibration parts of the machine. The increased masses should not exceed the permissible limits, exceeding which will lead to the economic inexpediency of this machine design $[3,5]$.

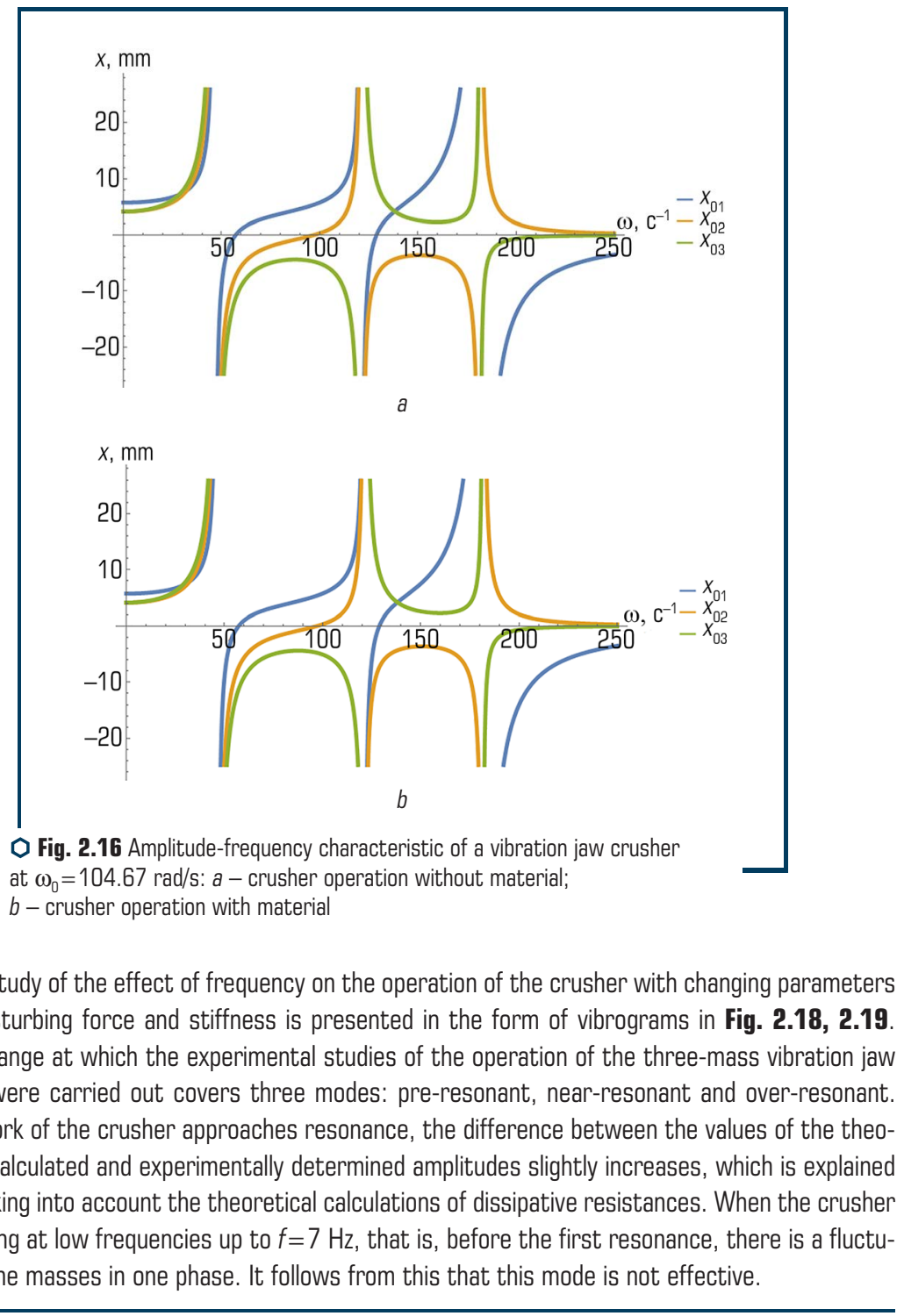




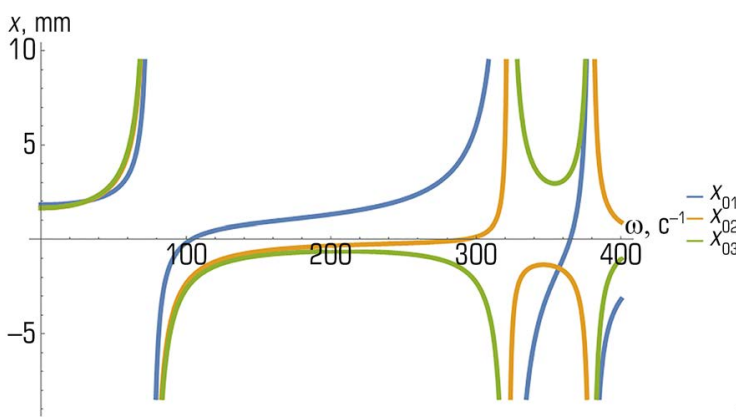

$a$

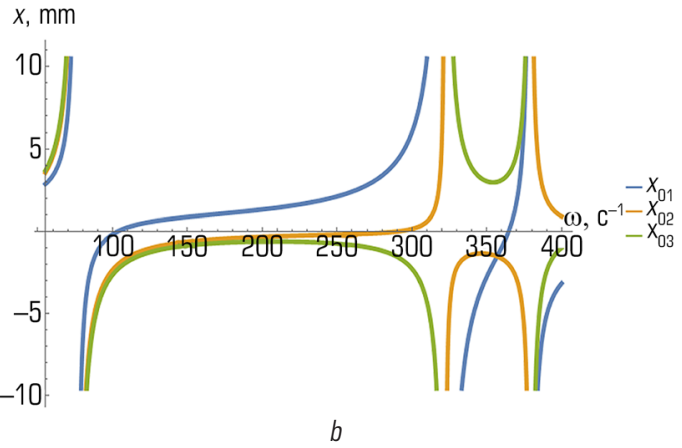

O Fig. 2.17 Amplitude-frequency characteristic of a vibration jaw crusher at $\omega_{0}=314 \mathrm{rad} / \mathrm{s}:$ a - crusher operation without material; $b$ - crusher operation with material

When the crusher is operating in the vicinity of a frequency of $12 \mathrm{~Hz}$, there is almost no crushing of the material. This can be explained by the fact that the vibrator does not create forces at a given frequency that could effectively destroy the material.

In the frequency range $14.5 \mathrm{~Hz}<f<26 \mathrm{~Hz}$, the material is efficiently comminuted. The efficiency of crushing is somewhat reduced when the crusher is operating in the vicinity of a frequency of $22 \mathrm{~Hz}$. This can be explained by a decrease in the amplitude of oscillation of the second and third masses and a decrease in vertical oscillations of the crusher body, which increases the residence time of the material in the crushed chamber.

When studying the influence of the stiffness of the elastic systems of the crusher on the crushing process, the following features were established. As the stiffness of the system increases, the effective range of the crusher shifts towards an increase in the angular frequency. This is due to the shift of the resonance point towards an increase in the angular frequency of the crusher vibrations. This property can be used to create a crusher control system. 

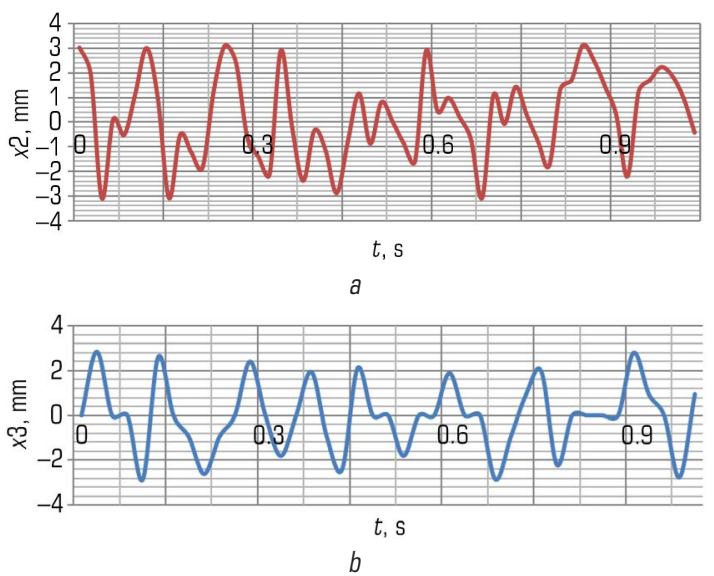

O Fig. 2.18 Vibrograms of vibrations of the masses of the crusher when working with material $\left(F=4111 \mathrm{~N}, c_{2}=219052 \mathrm{~N} / \mathrm{m}\right)$ :

$a$ - displacement of the second mass; $b$ - displacement of the third mass
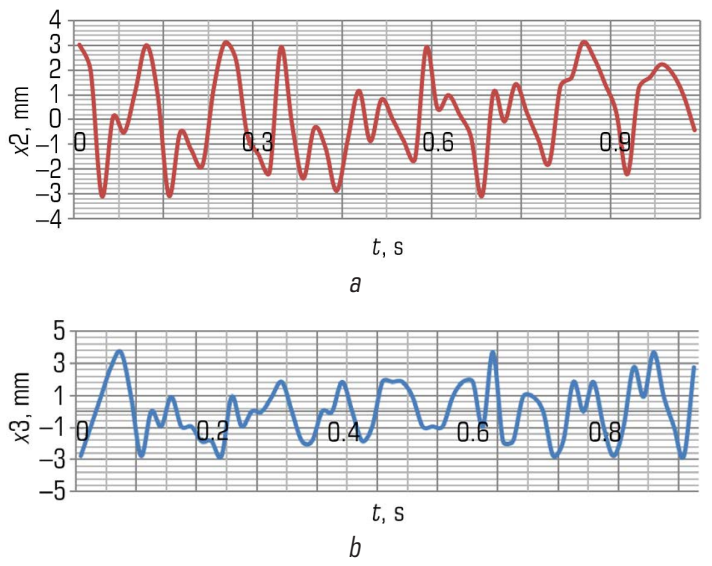

O Fig. 2.19 Vibrograms of vibrations of the crusher masses when working with material $\left(F=4111 \mathrm{~N}, c_{2}=234438 \mathrm{~N} / \mathrm{m}\right): a$ - displacement of the second mass; $b$ - displacement of the third mass

However, too much increase or decrease in stiffness adversely affects the performance of the machine. So, with a stiffness $\mathrm{N} / \mathrm{m}$, in the frequency range, the second and third masses move in phase, and the first in antiphase. Thus, the operation of the crusher at low frequencies is not 
efficient at a given stiffness. At very low stiffness, the efficiency of energy transfer from the vibrator to the third mass deteriorates, as evidenced by experimental data. The vibration amplitudes of the third mass, provided that $\mathrm{N} / \mathrm{m}$ are insignificant. The features of the impact of stiffness are as follows. As stiffness increases, the crusher's effective operating range shifts towards zero. If the stiffness is increased too much, the three-mass system turns into a two-mass one. With a decrease in stiffness, the range of effective operation of the crusher narrows, and in conditions of too large a decrease in stiffness, the system leaves the state of equilibrium, which has a negative effect on the crushing process.

\subsection{DISCUSSION OF RESEARCH RESULTS}

An efficient crusher operation can be achieved by operating it in the vicinity of resonance, i.e. at a high value of the coefficient of resonant amplification of oscillations. However, this mode is sensitive to changes in external influences on the workflow. It is possible to reduce external influences by automatically adjusting the operating mode of the machine.

The control of the vibration machine is to ensure the optimal operating mode, in which the specified displacements, speeds and accelerations are achieved [6-8]. The set values of displacements, speeds and accelerations of a vibration jaw crusher can be achieved both in pre-resonant and over-resonant operating modes.

The creation of a control system for the amplitude-frequency characteristic and taking into account the dependences of the moment of resistance on the frequency of the driving force underlies the method of regulating the working process of the crusher. This task can be achieved by correctly calculating the parameters of the three-mass vibration jaw crusher.

The second way to regulate the operating mode of a three-mass vibration crusher is to adaptively change the stiffness of the system. The third way to regulate the operating modes of the crusher is to change the vibration frequency.

On the basis of the above studies, algorithms were formulated for calculating the main parameters and controlling the working process of a vibration three-mass jaw crusher (Fig. 2.20, 2.21), respectively.

A physical model and a mathematical model of a vibration jaw crusher have been developed, taking into account the elastic - inertial properties, power and geometric characteristics of the «jaw - fractional material» system.

One of the main research tools were the amplitude - frequency characteristics of the system movement. The studies have established the regularities of the change in the elasticity coefficients depending on the frequency, the coefficients that determine the rational ratios of the vibration masses.

The optimal values of the amplitude of the oscillations of the masses have the greatest values, provided that the coefficient of regulation of elasticity in frequency $n$ is in the range of $0.6 \leq n \leq 1$. Within the frequency range of $14.5 \mathrm{~Hz}<f<26 \mathrm{~Hz}$, material is effectively crushed. The numerical values of the coefficient $k_{23}$ are established, which determine the rational ratios of vibration masses. 
The studies carried out and their results indicate that the main stresses causing the destruction of the material are shear stress. With an increase in the size of the major semiaxis of the crack ellipse, the total stresses decrease, which indicates the destruction of the brittle body. However, the stresses $\sigma \rho$ acting along the radius slightly increase. The graphs (Fig. 2.4) reflect the picture of the stress distribution in a spherical body relative to the point of application of the force along the angle $\varphi$. The graph (Fig. 2.5) shows the change in normal stresses depending on the distance $r$. Analysis of the graph (Fig. 2.6) makes it possible to conclude that at $r->0$, the stresses increase indefinitely. As a result of this, the stresses and strains in the cracked body must be determined in the vicinity of the center of the body. In addition, it was found that the complete elliptical integral of the second kind has an insignificant effect on the change in the stress pattern in the body. It should be noted that when solving the problem using the Legendre functions, 15 constants appear in the stress equations, which together have a significant effect on the stress pattern in the body. These constants were a numerous method based on the equations of the potential energy of a deformed body.

Equations (2.21), (2.23) and (2.30) fully reflect the picture of energy consumption for material destruction. Such studies are planned as a continuation of the topic under consideration through experimental studies and consideration of several members of the series and optimization of the parameters of crushing machines. The proposed approach to studying the energy characteristics of material destruction in the crusher chamber with a guaranteed zone of stability of parameters in the resonance zone can be used for other processes. Such processes include the destruction of materials during dynamic cutting of soils, processing of materials in mills and sorting of materials, which are gaining widespread use in various European countries.

\section{CONCLUSIONS TO SECTION 2}

1. A physical model and a mathematical model of a vibration jaw crusher have been developed.

2. The stress-strain state of the material in the process of its destruction for a continuous medium and surrounded by an existing crack has been investigated.

3. The efficiency of the crusher's working process is proved by the practical achievement of its work in the vicinity of resonance. That is, at a high value of the resonant amplification factor of the oscillations.

4. It has been determined that the optimal values of the amplitude of the oscillations of the masses have the greatest values, provided that the coefficient of regulation of elasticity in frequency $n$ is within $0.6 \leq n \leq 1$. Within the frequency range of $14.5 \mathrm{~Hz}<f<26 \mathrm{~Hz}$, material is effectively crushed.

5. The proposed approach to study the energy characteristics of material destruction in the crusher chamber with the provision of a guaranteed zone of stability of parameters in the resonance zone.

6. The studies carried out and their results indicate that the main stresses that lead to the destruction of the material are shear stress.

7. Algorithms for calculating the parameters of a vibration three-mass crusher have been formulated. 


\section{REFERENCES}

1. Baladinskyi, V. L., Nazarenko, I. I., Onyshchenko, O. H. (2002). Budivelna tekhnika. Kyiv - Poltava: KNUBA-PNTU, 463.

2. Nazarenko, I. I. (2010). Prykladni zadachi teorii vibratsiinykh system. Kyiv: Vydavnychyi Dim "Slovo", 440.

3. Mishchuk, Y., Nazarenko, I., Mishchuk, D. (2021). Definition of rational operating modes of a vibratory jaw crusher. Naukovyi Visnyk Natsionalnoho Hirnychoho Universytetu, 4, 56-62. doi: http://doi.org/10.33271/nvngu/2021-4/056

4. Nazarenko, I., Mishchuk, Y., Mishchuk, D., Ruchynskyi, M., Rogovskii, I., Mikhailova, L. et. al. (2021). Determiantion of energy characteristics of material destruction in the crushing chamber of the vibration crusher. Eastern-European Journal of Enterprise Technologies, 4 (7 (112)], 41-49. doi: http://doi.org/10.15587/1729-4061.2021.239292

5. Nazarenko, I., Mishchuk, E., Kuchinsky, V. (2019). Assessment and analysis of basic design the cone crushers. Girnichi, budivelni, dorozhni ta meliorativni mashini, 94, 5-15. Available at: http://gbdmm.knuba.edu.ua/article/view/216440

6. Lapin, R., Kuzkin, V. (2019). Calculation of the normal and shear compliances of a three-dimensional crack taking into account the contact between the crack surfaces. Letters on Materials, 9 (2), 234-238. doi: http://doi.org/10.22226/2410-3535-2019-2-234-238

7. Zou, J., Han, J., Yang, W. (2020). Investigating the Influences of Indentation Hardness and Brittleness of Rock-Like Material on Its Mechanical Crushing Behaviors. Mathematical Problems in Engineering, 2020. doi: http://doi.org/10.1155/2020/4713532

8. Nazarenko, I., Gaidaichuk, V., Dedov, O., Diachenko, O. (2018). Determination of stresses and strains in the shaping structure under spatial load. Eastern-European Journal of Enterprise Technologies, 6 (7 (96)), 13-18. doi: http://doi.org/10.15587/1729-4061.2018.147195

9. Nazarenko, I. I., Ruchynskyi, M. M., Sviderskyi, A. T., Kobylanska, I. M., Harasim, D., Kalizhanova, A., Kozbakova, A. (2019). Development of energy-efficient vibration machines for the buiding-and-contruction industry. Przeglad Elektrotechniczny, 95 (4), 53-59. doi: http://doi.org/ 10.15199/48.2019.04.10

10. Nazarenko, I., Svidersky, A., Kostenyuk, A., Dedov, O., Kyzminec, N., Slipetskyi, V. (2020). Determination of the workflow of energy-saving vibration unit with polyphase spectrum of vibrations. Eastern-European Journal of Enterprise Technologies, 1 (7 (103)), 43-49. doi: http:// doi.org/10.15587/1729-4061.0.184632

11. Vasiliev, L. M., Vasiliev, D. L., Malich, M. G. (2021). Modeling the process of disintegration of solid materials by asymmetric loading in crushing machines in order to find ways to reduce energy costs. Energy- and resource-saving technologies of developing the raw-material base of mining regions. Petroșani: UNIVERSITAS Publishing, 457-473. doi: http://doi.org/10.31713/m1028

12. Hong, S. J., Yang, H. J. (2019). A Study on the Impact Load Quantification of the Jaw Crusher. Journal of Drive and Control, 16 (2), 1-7. doi: https://doi.org/10.7839/KSFC.2019.16.2.001 
13. Blokhin, V. S., Bolshakov, V. I., Malich, N. G. (2006). Osnovnye parametry tekhnologicheskikh mashin. Mashiny dlia dezintegratsii tverdykh materialov. Part. I. Dnepropetrovsk: IMA-press, 404.

14. Vaisberg, L. A., Zarogatskii, L. P., Turkin, V. la. (2004). Vibratsionnye drobilki. Osnovy rascheta, proektirovaniia i tekhnologicheskogo primeneniia. Saint Petersburg: Izd-vo VSEGEl, 306.

15. Nazarenko, I., Dedov, O., Bernyk, I., Rogovskii, I., Bondarenko, A., Zapryvoda, A. et. al. (2020). Determining the regions of stability in the motion regimes and parameters of vibratory machines for different technological purposes. Eastern-European Journal of Enterprise Technologies, 6 (7 (108)), 71-79. doi: http://doi.org/10.15587/1729-4061.2020.217747

16. Nesterenko, M., Nazarenko, I., Molchanov, P. (2018). Cassette Installation with Active Working Body in the Separating Partition. International Journal of Engineering \& Technology, 7 (3.2), 265-268. doi: http://doi.org/10.14419/jijet.v7i3.2.14417

17. Bernyk, I., Luhovskyi, O., Nazarenko, I. (2018). Effect of rheological properties of materials on their treatment with ultrasonic cavitation. Materiali in Tehnologije, 52 (4), 465-468. doi: http://doi.org/10.17222/mit.2017.021

18. Nazarenko, I., Gavryukov, O., Klyon, A., Ruchynsky, N. (2018). Determination of the optimal parameters of a tubular belt conveyor depending on such an economical. Eastern-European Journal of Enterprise Technologies, 3 (1 (93)), 34-42. doi: http://doi.org/10.15587/17294061.2018.131552 